\section{THE USES OF BLEEDING IN INFLAMMATION AND OTHER DISEASES.}

By John Hughes Bennett, M.D., F.R.S.E., Professor of the Institutes of Medicine, and of Clinical Medicine, in the University of Edinburgh.

The admirable paper read by Dr. Markham to the MedicoChirurgical Society of London, and inserted in two recent numbers of this Journal (April 9th and 16th), appears to me deserving of special notice. I have little doubt that he has correctly stated what ought to constitute one of the indications for bleeding in diseases.

Twenty years ago, it was supposed that the primary cause of inflammations was an increased action of the vessels, but more especially of the arteries, which sent forwards or determined to the parts a greater amount of blood than the veins could carry away. As a result, the smaller vessels became blocked up and distended, while the fluid part or lymph was squeezed through their walls, and infiltrated into the surround ing tissues. The remedy for this was the removal of blood from the part, either by local or general bleeding, by which it was thought this condition could be at once removed. But it is now understood that not only is this theory wrong, but facts have shown that the practice which flows from it is not effective. Instead of the morbid action beginning in the vessels, it truly begins in the tissues outside the vessels. These, in health, are continually attracting and selecting from the blood such of its constituents as are necessary for their own nourish ment. Thus the cells of the liver select what is necessary to form bile; the salivary glands what is necessary to form saliva; the muscles what is required to form muscle; and bone what is wanted to form bone. If these parts are injured, healthy nutrition does not take place; but instead of it there is attracted from the blood that lymph, or fluid part of the blood, which is so essential a feature in all inflammation. Instead of being pushed out by a vis a tergo, it is drawn out by a vis a fronte-the active force causing the disturbance is not in the vessel or in the blood, but in the cells and tissues to which these are distributed.

Again, when the living fluid part of the blood has in this way got outside the vessel, a wonderful new growth of cells takes place in it, whereby what is unnecessary is removed by absorption and excretion, and what is required is developed into new texture. This stage of the process is accompanied by more or less excitement; an increased flow of blood is drawn to the part; more nourishment is required, and nature supplies it. In the same manner that when the leaves bud in spring, more sap exists in and is drawn from the vessels in plants; that during the annual growth of the stag's horn, all the vessels in the neighbourhood are turgid with blood; that when new teeth appear in the infant, the gums are red and tender; that when, in short, all active processes of local growth are proceeding, the pulse is strong, the blood flows with increased velocity, and the neighbouring tissues are congested so, for the wisest and best purposes, does nature set up a similar action in inflammation, and turns to use that exudation which has been poured out, by transforming it into cells.

According to this theory, we must look upon such excitement in the neighbourhood of an inflamed part as salutary, whereas formerly it was regarded with apprehension. The increased force of the pulse was thought dangerous, and the rapid flow of blood which used to be looked upon as injurious to the in. dividual we now regard as necessary for a cure. Instead, therefore, of diminishing or lessening these useful changes by blood-letting and antiphlogistics, it is clear that theoretically they ought to be left undisturbed, and even supported when necessary. The correctness of this theory has been proved by actual experiment and experience on a large scale, a marked improvement in the recovery from severe inflammations having everywhere resulted from abandoning a lowering treatment in that class of diseases. Inflammations of the lungs, of the pericardium, of the pleura, and so on, have been proved not only to be much less fatal, but to get well in a much shorter time, since bleeding and antiphlogistics were replaced by nutrients and stimulants.

But this is not saying that blood-letting can never be useful in diseases, and may not occasionally be beneficial even in inflammations. Thongh it has been industriously circulated that I am opposed to bleeding under all circumstances; so far is this from being the case, that I have carefully maintained the contrary. Thus, in my original paper in the Edinburgh Monthly Journal for February 1857, pp. $782-3$, I observe :"But whilst large and repeated bleedings, practised with a view of arresting the disease, appear to me opposed to a correct pathology, small and moderate bleedings, directed to palliate certain symptoms, and especially excessive pain and dyspncea, may reasonably be had recourse to, and unless there be great weakness, without any fear of doing injury. I have often been struck, especially in cases where large thoracic aneurisms cause these symptoms, with the small loss of blood which will occasion marked relief. The same result may be hoped for in other cases where the congestion is passive, even when that is associated with active repletion of blood, followed by exudation. Dut I need scarcely remark, that this mere palliative object of blood-letting is not the ground on which the practice has hitherto been based, and that in this point of view it requires to be very differently explained." Again, at page 795 , I say:- "There are cases, which were formerly" mistaken for inflammation, in which blood letting may still be useful. I allude to those where an obstruction to the circulation exists in the heart and lung, dependent on over-distension of the right side of the former organ, and cases of venous congestion, engorgement, and perhaps œdema of the latter; also, certain cases of bronchitis preventing aëration, of aneurisms, and of asphyxia. Although even here the true value of the remedy has yet to be positively ascertained, the special cases demanding it more carefully discriminated, and the mechanical principles which justify the practice determined." These same passages are given verbatim in the second and third editions of my Clinical Lectures on the Principles and Practice of Medicine.

It follows that, in the very class of cases in which Dr. Narkham says he has bled with advantage, I have always maintained the utility of employing general blood-letting with moderation. So with topical blood-letting, wherever it can directly operate on the inflamed or congested part, it may be beneficial, as in certain external inflammations, conjunctivitis, or hæmorrhoids; but in deep-seated internal inflammations it can be of little benefit. This is a widely different method of employing the remedy from what was formerly practised; viz., by repeated large bleedings, which lowered the pulse and exhausted the patient, at a time when he required all his strength and vigour to support the new changes in growth required by the economy. I believe that an equally good effect would have resulted in some of Dr. Markham's cases, from the extraction of one-half or even one-fourth of the amount of blood he took away. So far, however, is the proper use of blood-letting as a palliative in inflammation, and as a curative measure in congestion of the heart, asphyxia, and so on, opposed to pathology, that it is quite in harmony with it; and, in all these cases, its good effects fully explain the benefits, temporary and permanent, which have been recorded in its favour by practitioners both ancient and modern.

\section{ON TIIE CAUSES OF PULMONARY C O N S U M P'T I O N .}

By James Tunnbuli, M.D., Physician to the Liverpool Royal Infirmary.

[Concluded from page $\mathbf{3} 6 \mathrm{i}$.]

Occupations producing Inhalation of Irritating Particles. There are many occupations, which, it can be shown, have a direct tendency to cause chronic pulmonary disease by the local irritation they produce on the lungs, in addition to the injurious effect which is often, at the same time, caused by the sedentary nature of the employment itself. It has long been known that stone masons, who are engaged in cutting stones, suffer from inhalation of the silicions particles, and become affected with pulmonary disease; and also that the grinders employed in manufacturing cutlery suffer in a similar way, especially when the processes are carried on in confined workshops, and without the aid of water, which prevents the diffusion of the silicious and metallic particles in the atmosphere. Under the head of metal manufacture, Dr. Greenhow has given some statistics which prove very clearly the injurious influence of such employments; and, in respect to the operatives of Sheflield and Jirmingham, it is made apparent by the great divergence of the pulmonary death-rates in the male and female population. The men, being almost exclusively engaged in such employments, suffer most; and in Sheffield we find that the male pulmonary death-rate is 839 , whilst the female is only $6 \pi(0$; and in 